MQ NMR and SPME analysis of nonlinearity in the degradation of a filled silicone elastomer

S. C. Chinn, C. T. Alviso, E. S. Berman, C. A. Harvey, R. S. Maxwell, T. S. Wilson, R. Cohenour, K. Saalwachter, W. Chasse

October 16, 2008

Journal of Physical Chemistry B 
This document was prepared as an account of work sponsored by an agency of the United States government. Neither the United States government nor Lawrence Livermore National Security, LLC, nor any of their employees makes any warranty, expressed or implied, or assumes any legal liability or responsibility for the accuracy, completeness, or usefulness of any information, apparatus, product, or process disclosed, or represents that its use would not infringe privately owned rights. Reference herein to any specific commercial product, process, or service by trade name, trademark, manufacturer, or otherwise does not necessarily constitute or imply its endorsement, recommendation, or favoring by the United States government or Lawrence Livermore National Security, LLC. The views and opinions of authors expressed herein do not necessarily state or reflect those of the United States government or Lawrence Livermore National Security, LLC, and shall not be used for advertising or product endorsement purposes. 


\title{
MQ NMR and SPME analysis of nonlinearity in the degradation of a filled silicone elastomer
}

\author{
Sarah C. Chinn*1, Cynthia T. Alviso ${ }^{1}$, Elena S.F. Berman ${ }^{1 *}$, \\ Christopher A. Harvey ${ }^{1}$, Robert S. Maxwell ${ }^{1}$, Thomas S. Wilson ${ }^{1}$, Rebecca Cohenour ${ }^{2}$, \\ Kay Saalwächter ${ }^{3}$, Walter Chassé ${ }^{3}$ \\ ${ }^{1}$ Lawrence Livermore National Laboratory \\ 7000 East Ave, Livermore, CA 94550 \\ ${ }^{2}$ Honeywell Inc. Federal Manufacturing \& Technologies Kansas City Plant \\ Kansas City, MO 64141 \\ ${ }^{3}$ Institut für Physik, Martin-Luther-Universität Halle-Wittenberg \\ Betty-Heimann-Str. 7, D-06120, Halle, Germany \\ *Current address: Los Gatos Research, 67 East Evelyn Ave. Ste. 3, \\ Mountain View, CA 94041
}




\section{Abstract}

Radiation induced degradation of polymeric materials occurs via numerous, simultaneous, competing chemical reactions. Though degradation is typically found to be linear in adsorbed dose, some silicone materials exhibit non-linear dose dependence due to dose dependent dominant degradation pathways. We have characterized the effects of radiative and thermal degradation on a model filled-PDMS system, Sylgard 184 (commonly used as an electronic encapsulant and in biomedical applications), using traditional mechanical testing, NMR spectroscopy, and sample headspace analysis using Solid Phase Micro-Extraction (SPME) followed by Gas Chromatography/Mass Spectrometry (GC/MS). The mechanical data and ${ }^{1} \mathrm{H}$ spin-echo NMR indicated that radiation exposure leads to predominantly crosslinking over the cumulative dose range studies ( 0 to $250 \mathrm{kGray)}$ with a rate roughly linear with dose. ${ }^{1} \mathrm{H}$ Multiple Quantum NMR detected a bimodal distribution in the network structure, as expected by the proposed structure of Sylgard 184. The MQ-NMR further indicated that the radiation induced structural changes were not linear in adsorbed dose and competing chain scission mechanisms contribute more largely to the overall degradation process in the range of 50 - 100 kGray (though crosslinking still dominates). The SPME-GC/MS data were analyzed using Principal Component Analysis (PCA), which identified subtle changes in the distributions of degradation products (the cyclic siloxanes and other components of the material) as a function of age that provide insight into the dominant degradation pathways at low and high adsorbed dose.

* Corresponding author: Email: chinn7@,1ln1.gov; Phone: 925-422-5514 


\subsection{Introduction:}

Due to the chemical and physical nature of the polydimethylsiloxane backbone, silicone polymeric networks have served as near ideal model systems for studying the structural influence on the polymer chain dynamics, and thus the mechanical properties of broad classes of polymer-filler composite systems, including micro and nanocomposites. With applications ranging from cushions, medical devices, lithographic materials, insulators, and coatings, studies of filled silicone elastomers comprise a large amount of the polymer literature due to their versatility and ubiquitous presence in the industrial world. Though the effects of ionizing radiation on silicone polymers has been studied for over 50 years, there are still unresolved issues. ${ }^{1-5}$ Most work to date has studied ranges of adsorbed dose where the effects on molecular weight, sol-fraction, evolved gas, etc. are typically found to be linear with dose. Further, the significant fraction of these studies have been performed on soluble polymer chains and not crosslinked networks. In many cases, where systems were studied past their gel point, insight into the effects of radiation typically have come from study of the soluble fraction remaining and not direct study of the gel, or crosslinked fraction.

Similar to most other polymeric materials, silicone based material degradation generally occurs by multiple competing chain breaking (scission, unzipping, backbiting) or chain formation mechanisms (i.e. H vs. Y crosslinking via varying free radical mechanisms), bonding to the filler matrix, etc. ${ }^{1-4,6-15}$ For both thermally and radiation induced degradation multiple, competing reaction pathways can exist and occur simultaneously to varying extent dependent on variables such as total input energy, monomer types (i.e. stabilizing phenyl groups), additives (fillers, stabilizers, etc.), and 
network topology. The resulting effects on the polymer are the net effect of the reactions that have occurred. Thermal degradation typically follows standard kinetics of thermally activated processes though with the complications caused by multiple allowable reaction pathways. In fact, experiments have observed a switch over between dominant mechanisms previously (e.g. dominance of acid-catalyzed hydrolytic chain scission at low temperatures $\left(<110^{\circ} \mathrm{C}\right)$ and backbiting of free chain ends to form cyclic siloxanes at high temperatures (typically greater than $120{ }^{\circ} \mathrm{C}$ ). ${ }^{10-13,16}$ Radiation damage, however, has generally observed to be linear in adsorbed dose over distinct dose ranges that depend on the structure and composition of the polymer composite, though a few studies have observed non-linear behavior at sufficiently high adsorbed dose. Over extended dose ranges the competition between these overlapping reactions can, however, lead to nonlinear behavior. ${ }^{1,2,4,6,17}$ Unfortunately, the specific changes in the dominating mechanisms that may be responsible for this variance, particularly in radiation induced degradation, is generally poorly understood. This is due in no small part to a lack of methodologies that provide correlation between changes in chemical speciation and the remaining composite network structure that determine the engineering properties.

Here we describe a general multipronged approach to developing improved insight into the chemical origins of degradation in a commercial silica filled silicone polymer formulation that is broadly applicable to silicone based materials. In this study the effects of gamma radiation and exposure to elevated temperature are investigated as test cases using a combination of advanced ${ }^{1} \mathrm{H}$ Multiple Quantum (MQ) NMR and SPME-GC/MS. The benefits of MQ-NMR spectroscopy lie in its ability to identify network specific changes that are not available by other means and enable the 
quantitative assessment in structural dynamics as a function of long-term exposure to chemically, thermally, or radioactively harsh environments. ${ }^{6-9}$ The MQ-NMR growth curves were analyzed with the aid of new robust algorithms that drastically reduce the variability in analyzing the results. Further, SPME-GC/MS has found great use in the identification of volatile offgassing components ${ }^{18,19}$ and previously has been used to identify the major byproducts of siloxane degradation ${ }^{8,14,20}$ thus aiding in the elucidation of degradation mechanisms. And though the combination of SPME-GC/MS data sets with modern multivariate analysis methods has been extensively in the food industry, ${ }^{21-24}$ its applicability to SPME analysis of silicone degradation has not been reported. Here, the GC/MS chromatograms were analyzed by Principal Component Analysis to determine common signatures of aging mechanisms and to gain a complete understanding of degradation prevalence and pathways. Combined with traditional analysis, the MQ-NMR and SPME-GC/MS results allowed the quantification of changes in the network structure and identification of responsible degradation mechanisms as a function of cumulative gamma radiation dose or temperature.

\subsection{Experimental:}

\subsection{Sample preparation}

Experiments were performed on samples of Sylgard 184, which due to its versatile mechanical, chemical, and dielectric properties, has a number of other common uses that include biomedical MEMS devices, aerospace sealants, and use as a soft lithography material. Sylgard 184 is a platinum catalyzed vinyl addition silicone polymer manufactured by Dow Corning. The commercially available mixture consists of a 10:1 
mixture of Sylgard 184 base resin and curing agent consisting of polymethylhydrosiloxane (PMHS) and polydimethylsiloxane (PDMS). The base resin is composed of $\sim 65 \%$ vinyl end-capped PDMS and $\sim 35 \%$ soluble silica filler $\left(50 \% \mathrm{SiO}_{2}\right.$, 45\% trimethyl end-capped PDMS, and 5\% vinyl end-capped PDMS) plus $\sim 6$ ppm chloroplatinic acid adduct as a catalyst. The samples were vacuum de-aired and cured for seven days at room temperature.

Test samples were cut into strips and placed into aging containers that were either left open to air or back-filled with nitrogen. The samples were then exposed to ${ }^{60} \mathrm{Co}$ radiation at a dose rate of $0.5 \mathrm{kGy} / \mathrm{hr}$ for times necessary to reach desired doses ranging from 10 to $250 \mathrm{kGy}$. After aging, samples were further prepared and characterized as detailed below.

\subsection{Mechanical and rheological testing}

A TA Instruments Q1000 Differential Scanning Calorimeter (DSC) was used to determine glass transition temperatures. Approximately 10-15 mg of sample was sealed in an aluminum pan. The samples were subjected to a modulated $1.5^{\circ} \mathrm{C} / \mathrm{min}$ temperature ramp from $-160{ }^{\circ} \mathrm{C}$ to $50{ }^{\circ} \mathrm{C}$ with a temperature modulation of $+/-0.27{ }^{\circ} \mathrm{C}$ every 20 seconds under a helium purge with a flow rate of approximately $50 \mathrm{~mL} / \mathrm{min}$.

Young's moduli for the Sylgard 184 material were determined using an MTS Synergie 400 Tensile test frame equipped with a $50 \mathrm{~N}$ load cell. Test specimens were cut into strips with dimensions of approximately $30 \mathrm{~mm}$ long, $5 \mathrm{~mm}$ wide, and 1.5 to $2.2 \mathrm{~mm}$ thick. The specimen clamps were screw type and strain was measured based on grip separation, which limited measured properties to initial modulus. Testing was carried out 
using an initial gage length of $15 \mathrm{~mm}$ and at an initial strain rate of $0.5 \mathrm{~min}^{-1}$, consistent with ASTM D882-02. Reported results are the average of three or four tests per condition.

\subsection{NMR experiments}

\subsubsection{Correlation to structural parameters}

The residual dipolar couplings $<\Omega_{\mathrm{d}}>$ are a result of incomplete motional averaging of magnetic dipole-dipole interactions due to structural variables within the polymer that lead to inhomogeneities in the motion of the monomers and prevent complete averaging to zero of the dipolar interactions. The correlation between residual dipolar couplings measured by NMR methods and structural variables have been reviewed in detail elsewhere and can be summarized in the following correlation between residual dipolar couplings, the dynamic order parameter, $S_{b}$, and the number of statistical segments, $\mathrm{N}$, between constraints: ${ }^{25-30}$

$$
S_{b}=\frac{1}{P_{2}(\cos \alpha)} \frac{\left\langle\Omega_{d}\right\rangle}{\left\langle\Omega_{d}\right\rangle_{\text {static }}}=\frac{3 r^{2}}{5 N}
$$

Here the static dipolar coupling, $\left\langle\Omega_{d}\right\rangle_{\text {static }}$, is preaveraged by the rotation of the methyl groups in PDMS, $\mathrm{P}_{2}(\cos \alpha)$ is the second order Legendre polynomial describing the orientational changes between the dipolar vector and the chain axis, and $r$ describes the distance relationship between the end-to-end vector, $\mathbf{R}$, from that of the unperturbed melt, $\mathbf{R}_{\mathrm{o}}: \mathbf{r}=\mathbf{R} / \mathbf{R}_{\mathbf{0}}{ }^{31}$

\subsubsection{NMR Methods}


NMR experiments were performed on a Bruker Avance spectrometer with a proton Larmor frequency of $400.13 \mathrm{MHz}$. Ninety degree pulse lengths of $\tau_{\mathrm{p}}=5.45 \mu \mathrm{s}$ and recycle delays of 6 seconds were used. Traditional spin echo experiments ${ }^{26,32}$ were fit to a bimodal distribution of decaying exponentials. The curves were characterized in all cases by a short $\mathrm{T}_{2}$ regime assigned to low-mobility network chains and a long $\mathrm{T}_{2}$ domain attributed to the non-network chains and chain-ends that are located far from topological constraints. In this report, we have used the $\mathrm{T}_{2}$ analysis primarily to obtain insight into the sol fraction. The long $\mathrm{T}_{2}$ fraction is actually made up of both the sol fraction and spins residing on the highly mobile ends of dangling chains - as discussed in Mayer et al, and Cohen-Addad, for example. ${ }^{26,33}$ Though a gross simplification, however, the relative amount of long $\mathrm{T}_{2}$ domains can be treated as proportional to the sol fraction, and relative fractional changes in the long $\mathrm{T}_{2}$ domains are thus related to the relative changes in sol fraction. ${ }^{2,4,8}$

Multiple quantum NMR experiments were performed as described by Saalwächter et al. ${ }^{31,34}$ using the generic pulse sequence to excite even-quantum coherences. The dipolar couplings contributing to the normalized DQ build-up curve were fit as described in detail by Giuliani et al., ${ }^{15}$, and Saalwächter et al. using an inverted Gaussian of the form

$$
I_{n D Q}\left(\left\langle\Omega_{d}\right\rangle ; \tau_{D Q}\right)=0.5 *\left(1-e^{-\frac{2}{5}\left\langle\Omega_{d}\right\rangle^{2} \tau_{D Q}^{2}}\right)
$$

where $<\Omega_{\mathrm{d}}>$ is the average dipolar coupling and $\tau_{\mathrm{DQ}}$ is the DQ mixing time described above. For bimodal distributions, a weighted sum of two inverted Gaussians in the form of Equation 3 was used. In this experiment, attempts to fit buildup curves to inverted Gaussians with distributions of coupling frequencies as described in earlier work ${ }^{15,31}$ 
yielded nonphysical results. Instead, the fast Tikhonov regularization software FTIKREG was used to invert the distribution integral to estimate the distribution of coupling frequencies for each sample using Equation 3 as a kernel function, as described previously, but with modifications as described in the next section. ${ }^{15,31,35}$

\subsubsection{Modified regularization process for $M Q$ - data analysis}

In the original version of the modified regularization program for the estimation of coupling distributions, originally published by Weese, ${ }^{35}$ eq. (3) was directly used as kernel function. ${ }^{31}$ This creates a problem in that the final normalized DQ intensity, which must always reach 0.5 in the long-time limit, had to be enforced by adding a few longtime datapoints with $I_{\mathrm{nDQ}}=0.5$ (particularly necessary since data are only fitted up to $I_{\mathrm{nDQ}}$ $=0.45$, see below). Since FTIKREG was initially conceived for inverse Laplace transformations (i.e., just a falling exponential as kernel function), it has a built-in option to enforce $I=0$ in the long-time limit. In order to use this option and obviate the use of an arbitrary number of additional points, the program now internally fits decay data of the form $0.5-I_{\mathrm{nDQ}}$ to a falling Gaussian, $0.5 \times e^{-\frac{2}{5}\left(\Omega_{d}\right\rangle^{2} \tau_{D Q}{ }^{2}}$. In addition, we have implemented a new empirical kernel function,

$$
y=0.5 \times\left(1-\exp \left(-\left(0.378 * 2 \pi\left\langle\Omega_{d}\right\rangle\right)^{1.5} \tau^{1.5}\right) \cos \left(0.583 * 2 \pi\left\langle\Omega_{d}\right\rangle \tau\right)\right)
$$

that includes the slight maximum observed in the normalized data and thus gives more reliable results for wider or multimodal distributions. Details will be reported in an upcoming publication.

We have also implemented changes in the error parameter that is required by FTIKREG. This parameter should reflect the constant, absolute-scale statistical error 
associated with the data points, and is needed for a proper initialization of the regularization parameter. It affects the smoothing of the data and the number of distribution modes extracted from the data; too low regularization parameters commonly lead to an "overinterpretation" of the result in terms of too many modes. This is problematic for two reasons. First, the error increases for longer times (as the intensities become low) due to the normalization procedure needed to remove relaxation effects, ${ }^{31}$ thus rendering it non-constant. Second, Equation 3 is only an approximation of true data, which exhibits a weak maximum in narrowly distributed systems. This is in fact why data are only fitted up to $I_{n D Q}<0.45$ (or, with our modified fit, down to 0.05 ), which is the region of good agreement. For broad distributions, the inability of Equation 3 to model the maximum of the subcomponents leads to underestimations of more weakly coupled subcomponents.

In order to select an error parameter that leads to a physically realistic distribution, the program now automatically varies the error parameter over a variable range, and outputs the distributions for each value, along with the corresponding $\chi^{2}$ value (mean squared deviation between data and fit). See Figure 1 (A and B) for the variation of $\chi^{2}$ with the given error parameter, and the resulting distributions. We take the most realistic distribution (shown in bold in Figure 1) as the one for the lowest error parameter, below which $\chi^{2}$ saturates at a nearly constant value. In most cases, much lower error values lead to multiply peaked distributions, which however do not lead to better overall fits and are consequently not necessarily physically meaningful.

\subsection{Solid Phase MicroExtraction-Gas Chromatography}


Carboxen/PDMS $(85 \mu \mathrm{m})$ SPME fibers were purchased from Supelco. SPME headspace vials $(20 \mathrm{~mL})$, crimp caps and septa $(20 \mathrm{~mm}$, teflon/blue silicone, level 4) were purchased from MicroLiter Analytical Supplies. Three samples, weighing approximately 20-30 mg each, were placed in $20 \mathrm{~mL}$ SPME headspace vials. One set of Sylgard 184 samples (pristine and irradiated) were analyzed as-is and one set was placed in a $70^{\circ} \mathrm{C}$ oven for two weeks.

Headspace SPME analyses for a given radiation dose and temperature were performed on a blank control vial and Sylgard 184. The samples were analyzed by SPME GC/MS using an automated system under the following conditions: $85 \mu \mathrm{m}$ Carboxen/PDMS SPME fiber, conditioned between samples for five minutes at $260^{\circ} \mathrm{C}$; headspace sampled at $50^{\circ} \mathrm{C}$ for 20 minutes and injected into the $\mathrm{GC}$ for one minute at $250^{\circ} \mathrm{C}$. The Agilent $6890 \mathrm{GC}$ was set for splitless injection and purged at 0.5 minutes using a J \& W Scientific DB-624 column (30 m, $0.25 \mathrm{~mm}$ ID, $1.4 \mu \mathrm{m}$ film) with a 1.0 $\mathrm{mL} / \mathrm{min}$ constant flow of helium. The 20 -minute run had the following temperature profile: $40^{\circ} \mathrm{C} / 1.05 \mathrm{~min} ., 23.41^{\circ} \mathrm{C} / \mathrm{min}$. to $260^{\circ} \mathrm{C}$, and held $6.81 \mathrm{~min}$. An Agilent 5973 mass spectrometer scanned the mass range from $35-450$ at a rate of $1.81 \mathrm{scans} / \mathrm{s}$ with no filament delay. Outgassing products were identified by comparison of their mass spectra to the NIST 02 mass spectral library.

\subsubsection{Principal Component Analysis}

PCA, a standard, unsupervised multivariate statistical technique, reduces a large data matrix to a few manageable variables that can be visualized and interpreted using a series of simple plots. ${ }^{36,37}$ PCA reduces the data complexity by calculating new variables 
called principal components, which represent linear combinations of the original variables and capture the greatest variation in the data set. The elution peaks and molecular fragments with the highest variance in intensity among the statistical groups identify important differences between samples.

GC-MS/MS spectra were aligned and analyzed using PLS Toolbox v. 4.1 (Eigenvector Research, Manson, WA) with MATLAB software v. 7.3 (MathWorks Inc., Natick, MA). The entire data set was first aligned to common peaks at 5.07, 6.75, 10.26, and 14.05 minutes utilizing a maximum allowable shift of 0.05 minutes for each of these reference peaks. Aligned individual spectra were normalized to unit area under the chromatogram, after which the entire data set was mean centered. PCA was performed to reduce the complexity of the data set and aid in the identification of chemical species that change significantly with treatment.

\subsection{Results \& Discussion:}

DSC, DMTA, and spin-echo NMR were applied to the pristine and irradiated samples. The glass transition temperature, the Young's modulus, and the transverse relaxation rate $\left(1 / T_{2}\right)$ for both the long $T_{2}$ and short $T_{2}$ domains were seen to increase near linearly with adsorbed dose (data in Table 1). These results indicate a decrease in mobility with increasing radiation due to the dominance of crosslinking reactions expected for silicone materials. ${ }^{2,438}$ Extracted NMR derived pseudo-sol fractions (see Experimental) decreased with increasing dose suggesting that radiation binds the small amount of sol component into the polymer network. The so-called Charlesby-Pinner plot of (Sol Fraction $+\sqrt{\text { Sol Fraction }})$ vs. 1/Dose $3^{39,40}$ is shown in Figure 2. The nonlinearity of 
the relationship is presumably due to the large degree of structural heterogeneity in the PDMS network, but might also reflect a transition in the dominant degradation mechanisms at about 50 kGray. ${ }^{39}$ Extrapolation of the data to infinite dose yields a ratio of $\mathrm{G}_{\mathrm{s}} / \mathrm{G}_{\mathrm{c}}=0.348$, where the $\mathrm{G}_{\mathrm{i}}$ value is the number of chain scission (s) or crosslinking (c) events per $100 \mathrm{eV}$ of energy absorbed, indicating that crosslinking is roughly three times as likely to occur as chain scission.

Multiple quantum growth curves were first fit to the discrete two-site model based on Equation 3. The curves fit moderately well to the two-site model, which supports the suggestion by Schmid and Michel that Sylgard 184 behaves like a bimodal network. ${ }^{41}$ The two modes can be attributed to long, mobile PDMS chains with lower values of residual dipolar couplings $(\sim 130 \mathrm{~Hz})$ and a mode comprised of short, less mobile PDMS chains, short hydrosilane crosslinkers, and polymer chains at or near the $\mathrm{SiO}_{2}$ filler surface, with higher $<\Omega_{\mathrm{d}}>$ values $(\sim 640 \mathrm{~Hz})$. The exact couplings and fractions of chains in Site 1 (low frequency coupling mode) are shown numerically in Table 1. With increasing dose, a general increase in both the low frequency and high frequency sites was observed, though the high frequency mode displayed some degree of nonlinearity in the increase possibly due to imperfections in the fit. The majority of the chains were found to be in Site 1 (low frequency coupling mode), and the overall fraction increased between 0 and $10 \mathrm{kGy}$ then decreased at higher doses. This suggests an initial softening of the material followed by a gradual stiffening of the network and indicates a competition of at least two degradation mechanisms as suggested in the Charlesby-Pinner analysis. The initial softening followed by stiffening has previously been observed in other filled silicone elastomers ${ }^{8}$ and has been attributed to an initial delamination of the 
polymer-filler interface due to a decrease in the hydrogen bonds that are responsible for the strong surface interaction. At higher doses, the subsequent decrease in chains in site 1 can be assumed to be due primarily to increased crosslinking.

The distribution of $<\Omega_{\mathrm{d}}>$ values from the FTIKREG analysis for each sample is shown in Figure 3 and the average $<\Omega_{\mathrm{d}}>$ value, $\chi^{2}$, and width of the primary coupling mode are shown in Table 1. This analysis resulted in primarily one distribution attributed to the large quantity of mobile polymer chains, with evidence of a shoulder due to the lower frequency coupling modes. The breadth of the distributions is ultimately due to the structural heterogeneity of the PDMS networks. The mean $\left\langle\Omega_{\mathbb{d}}>\right.$ was observed to increase slightly as a function of dose up to $50 \mathrm{kGy}$, followed by a larger jump to higher values at higher doses. The increase in $\left\langle\Omega_{\mathbb{d}}\right\rangle$ is due to an increase in average crosslink density of the material, again suggesting a dominance of radiation-induced crosslinking. The increase in the breadth of the distribution observed with increasing dose (simplistically calculated as the half width at half height of the $\left\langle\Omega_{\mathbb{d}}\right\rangle$ distribution curve, shown in Table 1) is ultimately due to an increase in molecular weight distribution and thus structural heterogeneity, which has been suggested to result from increased chain scission events at higher dose. ${ }^{15,39}$ Additionally, the asymmetry in the distribution is due to an increase in the high molecular weight component that cannot be separated from the low molecular weight component due to the structural heterogeneity of these materials. However, the relative width, defined as the width divided by the mean $\left\langle\Omega_{\mathrm{d}}>\right.$, appears to decrease up to $50 \mathrm{kGy}$ then increase at higher doses due to the increasing asymmetry of the distributions. The competition of mechanisms is manifest in the combined increase in 
mean $<\Omega_{\mathrm{d}}>$ and the broadening of the distribution width, though the data suggests that crosslinking is the dominant mechanism.

To obtain more information about the chemical nature of the changes observed as a function of radiation, headspace analysis was performed on the material. SPMEGC/MS analysis of the irradiated and thermally aged samples showed subtle trends in the total ion chromatographs, as demonstrated in Figure 4A for the irradiated samples and Figure $4 \mathrm{~B}$ for the irradiated samples further aged at $70^{\circ} \mathrm{C}$ for two weeks. The major peaks are identified according to Table 2. Small changes in peak intensity can be seen for a few select peaks but a detailed quantitative inspection of these changes would be difficult by traditional inspection methods. Note that traditional gases that evolved during irradiation, namely hydrogen, methane, and ethane, ${ }^{1,3,5}$ are likely being produced but were not observed in our experimental design. The primary reasons are because they pass through the column too rapidly to be detected and, since the evolved gases are not being trapped during irradiation (only the gases evolving from the bulk sample during post-irradiation analysis are being detected), the highly volatile, low molecular weight gas species are not, in fact, being sampled.

PCA of the irradiated samples that were analyzed without additional thermal aging is shown in Figure 5. Here, a plot of principal component 1 (PC1) vs. principal component 2 (PC2) reveals distinct groupings for samples irradiated at the different doses, indicating a discrete difference in offgassing signatures between the samples at each dose. Figure $6 \mathrm{~A}$ shows the scores plot for PC1, which constitutes $49.03 \%$ of the overall variance in the samples, and shows a trend of decreasing scores with increasing radiation up to $50 \mathrm{kGy}$ followed by a leveling off of the scores. The loadings plot in 
Figure 6B indicates that the THF peak at 5.43 minutes contributes most strongly to the positive scores (lowest doses), with a smaller contribution from the butyrolactone (peak at 8.6 minutes), a common oxidation product of THF. The negative scores are due primarily to the $\mathrm{d} 4-\mathrm{d} 7$ cyclic siloxanes (as well as residual peaks from the glass vials (present in the blank) at 2.7 minutes and trimethylsilanol at 5.08 minutes), which are more prevalent for samples at higher radiation doses. An additional peak from trans-(2chlorovinyl) dimethylethoxysilane at 6.91 minutes is presumed to be a reaction product from the catalyst and its presence at higher doses suggests a possible post-cure process occurring.

This analysis indicates that the changes in THF and butyrolactone concentration are most significant at low doses. The overall THF signal concentration decreased with increasing dose as can be seen by the slight decrease in signal intensity of the peak at 5.4 minutes in Figure 4A, though the overall effect is much more difficult to quantify on the total ion chromatogram (TIC). Between 10 and $50 \mathrm{kGy}$ there was a decrease in PC1 score that leveled off at higher doses. The negative scores seen here are primarily a result of changes in the $\mathrm{d} 4-\mathrm{d} 7$ cyclic siloxanes as well as contributions from the trimethyl silanol peak at 5.08 minutes. The sudden decrease in PC1 score between 10 and $50 \mathrm{kGy}$ as a result of the changes in cyclic siloxanes suggests an onset of backbiting chain scission reactions that could result either in additional crosslinking sites within the network or in the generation of cyclic siloxanes. The competing mechanisms and the marked change in PC1 score confirm the observations from the MQ NMR analysis.

Analysis of PC2 (29.07\% of the overall variance), shown in Figure 7 indicates a greater distinction between the behavior of the cyclic siloxanes. Positive loadings were 
present for the $\mathrm{d} 5$ and $\mathrm{d} 6$ cyclic siloxanes, with smaller positive loadings for the acetic acid and $\mathrm{d} 7$ and $\mathrm{d} 8$ cyclic siloxanes. The scores in Figure 7A increased with doses up to $50 \mathrm{kGy}$, and the corresponding loading data indicate that the $\mathrm{d} 5$ and $\mathrm{d} 6$ cyclic siloxanes were the largest contributors to this increase. A large change in behavior was seen between 50 and $100 \mathrm{kGy}$, where negative scores appeared, attributed mostly to the $\mathrm{d} 3$ and d4 cyclic siloxane, trimethyl silanol, residual glass, and the catalysis byproduct. Unfortunately, the imperfect spectral alignment evident in Figure $8 \mathrm{~B}$ hinders our ability to fully interpret the data, particularly for the $\mathrm{d} 6$ cyclic siloxane. However, the presence of the low molecular weight $\mathrm{d} 3$ and $\mathrm{d} 4$ cyclic siloxanes at the highest doses could be due to gamma irradiation initiated polymerization reactions known to occur in such systems. $^{42-44}$ Alternatively, the small amounts of chain scission reactions that are competing with the crosslinking reactions lead to backbiting reactions that form low molecular weight cyclic siloxanes. ${ }^{1}$

When the already irradiated samples were exposed to thermal degradation, a large amount of trimethyl silanol (peak at 5.06 minutes) was observed in the total ion chromatogram in Figure 4B. A scores plot of PC1 vs. PC2 for all doses at both temperatures, shown in Figure 8A, indicates that the increased temperature had a far larger effect on the samples than any of the irradiation dose. Interestingly, the variance in the data that can be attributed to the increased temperature was far greater than any variance attributed to the radiation alone. Figure $8 \mathrm{~B}$ shows the loadings plot for PC1, which represents $76.48 \%$ of the variance, with negative peak loadings correlated with room temperature aging and positive loadings correlated with $70^{\circ} \mathrm{C}$ aging. The large peak at 5.06 minutes is due to trimethylsilanol and was clearly the largest component that 
contributes to the differences in the TIC signatures. The presence of this peak indicates a thermally induced process involving the trimethyl silanol end groups, which are common products of hydrolytic chain scission. To a lesser extent, trans-(2-chlorovinyl)dimethylethoxy silane (peak at 6.91 minutes), the potential catalysis byproduct, appears to be more volatile at higher temperatures, as does a small amount of the $\mathrm{d} 8$ cyclic siloxane (14.05 minutes). The room temperature samples, mainly characterized as having negative PC1 scores and loadings, are associated with an acetic acid peak at 5.43 minutes and the remainder of the cyclic siloxanes. It is interesting to note that the presence of trimethyl silanol was significantly greater than the relative amounts of cyclic siloxanes, which are commonly generated from backbiting chain scission reactions. It is possible that the relatively low temperature of $70^{\circ} \mathrm{C}$ is not enough to significantly degrade the samples in the traditional mechanism, though it is more likely that the total contribution of chain scission mechanisms is simply producing smaller changes to the network structure than the removal of the trimethyl silanol groups.

The PCA analysis of the SPME/GC-MS data clearly indicates that in the dose range of $50 \mathrm{kGray}$ a change in the dominant mechanisms has occurred. At low doses, limited damage is being done to the network and the signatures observed are predominantly THF and THF oxidation products, though a number of cyclic siloxane and other synthesis by-products of incomplete cure chemistry are observed at lower doses. As the dose is increased, radiation induced chemistry begins to contribute significantly to the evolved gas speciation. These reactions include postcuring reactions, radiatively induced crosslinking reactions that cleave trimethyl silanol end groups, and radiative induced chain scission. As the crosslink density increases with increasing damage, the relative 
rates of the various cyclic siloxane species change, moving toward a preference for lower molecular weight cyclics, likely due to the increasingly restricted mobility of the network. The dramatic change observed in the in PC1 and PC2 scores between 50 and $100 \mathrm{kGy}$ occurs at the same doses in which the most significant change in both average residual dipolar coupling and distribution width occurred, again suggesting that the competition of crosslinking and chain scission mechanisms is dose dependent, with the dominance of crosslinking at the higher doses.

\subsection{Conclusions:}

A comprehensive approach involving mechanical, rheological, spectroscopic, and chromatographic analysis to determine both physical and chemical changes to PDMS networks as a function of degradation mechanisms was presented. The effects of oxidative gamma radiation on Sylgard 184 have been characterized with numerous analytical techniques to gain insight into the dependence on cumulative dose of the changes in segmental dynamics and mechanical properties of the material as well as the speciation of volatile and semi-volatile degradation products. Thermal analysis via DSC and DMTA indicated that there was a decrease in mobility as a result of radiationinduced crosslinking. NMR spin echo experiments detected decrease in sol fraction due to radiation-induced crosslinking. ${ }^{1} \mathrm{H}$ Multiple Quantum NMR provided increased insight into the degradation effects on network structure, revealing the competition between crosslinking and chain scission reactions. Headspace sampling from the bulk material followed by $\mathrm{GC} / \mathrm{MS}$ analysis was performed at room temperature and elevated temperature to identify changes in outgassing species as a function of dose. Subsequent 
PCA was performed to assist in the analysis of the subtle changes in the GC/MS data. While crosslinking was observed to dominate at all doses, at low dose ( $<50$ kGray), a mechanism that favored higher molecular weight cyclic species and low molecular weight degradation products was dominant. At higher doses, the competition between crosslinking and chain scission increased and backbiting reactions began to dominate, as reflected in the increased importance of lower molecular weight cyclic siloxanes. Further exposure to elevated temperature provides additional chain scission opportunities as evidenced by the significant offgassing of trimethyl silanol. Application of multivariate analysis afforded us the ability to distinguish between the contributions of postcure reactions and those of other degradation mechanisms and also indicated a change in dominant mechanisms at around 50 kGray consistent with the DSC and MQ-NMR, but not observed in the $\mathrm{T}_{2}$ and DMTA analysis. The PCA analysis of the SPME data and MQ-NMR have allowed for significantly enhanced insight into the chemical nature of radiation-induced degradation. Since typical predictions of long term behavior often rely on extrapolations of changes in mechanical properties with degradation, without specific connection to the complex chemical changes that are causing such changes, erroneous long-term predictions of material performance are likely. Improved insight, as demonstrated in this study, provides the opportunity to improve lifetime predictions.

\subsection{Acknowledgements:}

The authors wish to acknowledge Jason Giuliani and Erica Gjersing for assistance with the implementation of the MQ NMR experiments at LLNL and James Lewicki with assistance in the GC-MS data interpretation. We also gratefully acknowledge Theresa 
Morris for assistance with the DSC analysis at KCP. This work was performed under the auspices of the U.S. Department of Energy by Lawrence Livermore National Laboratory under Contract DE-AC52-07NA27344. 


\section{References}

(1) Aniruddha, S.; Clarson, S.; Widenhouse, C. J. Inorg. Organomet. Polym. 2008, 18, 207.

(2) Charlesby, A. Proc. Roy. Soc. London A 1955, 230, 120.

(3) Miller, A. J. Amer. Chem. Soc. 1960, 82, 3519.

(4) Charlesby, A.; Garratt, P. Proc. Roy. Soc. London A 1963, 273, 117.

(5) Miller, A. J. Amer. Chem. Soc. 1961, 83, 31.

(6) Maxwell, R.; Balazs, B. J. Chem. Phys. 2002, 116, 10492.

(7) Maxwell, R.; Cohenour, R.; Sung, W.; Solyom, D.; Patel, M. Poly. Deg. Stab. 2003, $80,443$.

(8) Chien, A.; Maxwell, R. S.; Chambers, D.; Balazs, B.; LeMay, J. J. Rad. Phys. Chem 2000, 59, 493.

(9) Maxwell, R.; Chinn, S. C.; Solyom, D.; Cohenour, R. Macromolecules 2005, 38, 7026.

(10) Grassie, N.; MacFarlane, I. Eur. Polym. J. 1978, 14, 875.

(11) Grassie, N.; MacFarlane, I.; Francey, K. Eur. Polym. J. 1979, 15, 415.

(12) Camino, G.; Lomakin, S.; Lazzari, M. Polymer 2001, 42, 2395.

(13) Camino, G.; Lomakin, S.; Lageard, M. Polymer 2002, 43, 2011.

(14) Hall, A.; Patel, M. Poly. Deg. Stab. 2006, 91, 2532.

(15) Giuliani, J. R.; Gjersing, E. L.; Chinn, S. C.; Jones, T. V.; Wilson, T. S.; Alviso, C. T.; Herberg, J. L.; Pearson, M. A.; Maxwell, R. S. J. Phys. Chem. B. 2007, 111, 12977.

(16) Patel, M.; Soames, M.; Skinner, A.; Stevens, T. S. Poly. Deg. Stab. 2004, 83, 111.

(17) Stevenson, I.; David, L.; Gauthier, C.; Arambourg, L.; Davenas, J.; Vigier, G. Polymer 2001, 42, 9287.

(18) Hakkarainen, M.; Groning, M.; Albertsson, A. C. J. Appl. Poly. Sci. 2003, 89, 867.

(19) Hakkarainen, M. Adv. Polym. Sci. 2008, 211, 23.

(20) Maxwell, R. S.; Chinn, S. C.; Alviso, C. T.; Harvey, C. A.; Giuliani, J. R.; Wilson, T. S.; Cohenour, R. Poly. Deg. Stab. 2009, 94, 456.

(21) Mildner-Szkudlarz, S.; Jelen, H. H. Food Chemistry 2008, 110, 751.

(22) Korhonova, M.; Hron, K.; Klimcikova, D.; Muller, L.; Bednar, P.; Bartak, P. Talanta 2009, 2009, 710.

(23) Mildner-Szkudlarz, S.; Jelen, H. H. J. Food Quality 2010, 33, 21.

(24) Jurado, J. M.; Ballesteros, O.; Alcazar, A.; Pablos, F.; Martin, M. J.; Vilchez, J. L.; Navalon, A. Analytical and Bioanalytical Chemistry 2008, 390, 961.

(25) Saalwächter, K.; Herrero, B.; Lopez-Manchado, M. Macromolecules 2005, 38, 9650.

(26) Cohen-Addad, J. P. Prog. NMR Spect. 1993, 25, 1.

(27) Callaghan, P. T.; Samulski, E. T. Macromolecules 2000, 33, 3795.

(28) Grinberg, F.; Kimmich, R.; Moller, M.; Molenberg, A. J. Chem. Phys. 1996, 105, 9657.

(29) Schneider, M.; Gasper, L.; Demco, D. E.; Blumich, B. J. Chem. Phys. 1999, 111, 402.

(30) Saalwächter, K. Prog. NMR Spect. 2007, 51, 1. 
(31) Saalwächter, K.; Ziegler, P.; Spyckerelle, O.; Haider, H.; Vidal, A.; Sommer, J.-U. J. Chem. Phys. 2003, 119, 3468.

(32) Mehring, M. Principles of High Resolution NMR in Solids; Springer-Verlag: Berlin, 1983.

(33) Mayer, B. P.; Chinn, S. C.; Maxwell, R. S.; Reimer, J. A. Chemical Engineering Science 2009, 64, 4684.

(34) Saalwächter, K. J. Chem. Phys. 2004, 120, 454.

(35) Weese, J. Computer Physics Communications 1992, 69, 99.

(36) Jolliffe, I. T. Principal Component Analysis; 2 ed.; Springer-Verlag: New York, 2002.

(37) Jackson, J. E. A User's Guide to Principal Components; John Wiley \& Sons: Hoboken, NJ, 2003.

(38) Dawes, K.; Glover, L. In Physical Properties of Polymers Handbook; Mark, J., Ed.; American Institute of Physics: Woodbury, NY, 1996, p 557.

(39) Saito, O.; Kang, H. Y.; Dole, M. J. Chem. Phys. 1967, 46, 3607.

(40) Charlesby, A.; Pinner, S. H. Proc. Roy. Soc. London A 1959, $249,367$.

(41) Schmid, H.; Michel, B. Macromolecules 2000, 33, 3042.

(42) Lawton, E. J.; Grubb, W. T.; Balwit, J. S. J. Polym. Sci. 1956, 19, 455.

(43) Wolf, C. J.; Stewart, A. C. J. Phys. Chem. 1962, 66, 1119.

(44) Chawla, A. S.; St. Pierre, L. E. J. Appl. Poly. Sci. 1972, 16, 1887. 


\section{Table Captions:}

Table 1. Results of DSC, DMTA, spin-echo NMR and two-site fit and FTIKREG analysis of MQ buildup curves.

Table 2. SPME-GC/MS Peak Assignments

\section{Figure Captions:}

Figure 1. (A) $\chi^{2}$ as a function of input error in FTIKREG program. (B) Distributions of $<\Omega_{\mathrm{d}}>$ as a function of input error (and $\chi^{2}$ ) for pristine Sylgard 184. The color of the line changes from black to lighter gray with increasing error. The arrows in both figures indicate the chosen $\chi^{2}$ value and distribution curve, with the appropriate distribution curve shown in bold.

Figure 2. Charlesby-Pinner analysis via spin echo NMR for irradiated Sylgard 184.

Figure 3. FTIKREG analysis of MQ growth curves for irradiated Sylgard 184.

Figure 4. GC/MS total ion chromatograms from SPME headspace analysis of samples irradiated and held at room temperature (A) and aged at $70^{\circ} \mathrm{C}$ for two weeks (B).

Figure 5. Principal Component Analysis of GC-MS data for samples irradiated and held at room temperature.

Figure 6. Scores (A) and loadings (B) plots for principal component 1.

Figure 7. Scores (A) and loadings (B) plots for principal component 2.

Figure 8. PCA plots comparing samples aged at $70^{\circ} \mathrm{C}$ vs. held at room temperature. (A) Scores plot for PC1 and PC2; (B) Loadings plot for PC1. Ovals are 90\% confidence ellipses. 
Table 1. Results of DSC, DMTA, spin-echo NMR and two-site fit and FTIKREG analysis of MQ buildup curves.

\begin{tabular}{|c|c|c|c|c|c|c|c|c|c|c|}
\hline \multirow{2}{*}{$\begin{array}{l}\text { Dose } \\
\text { (kGy) }\end{array}$} & \multirow{2}{*}{$\mathrm{T}_{\mathrm{g}}\left({ }^{\circ} \mathrm{C}\right)$} & \multirow{2}{*}{$\begin{array}{c}\mathrm{G}^{\prime} \\
(\mathrm{MPa})\end{array}$} & \multirow{2}{*}{$\begin{array}{c}1 / T_{2} \\
\left(\mathrm{~ms}^{-1}\right)\end{array}$} & \multicolumn{3}{|c|}{ 2-Site Fit } & \multicolumn{4}{|c|}{ FTIKREG } \\
\hline & & & & $\begin{array}{c}\left\langle\Omega_{\mathrm{d}}>_{1}\right. \\
(\mathbf{H z})\end{array}$ & $\begin{array}{c}<\Omega_{\mathrm{d}}>_{2} \\
(\mathrm{~Hz})\end{array}$ & $\mathrm{X}_{1}(\%)$ & $\begin{array}{l}<\Omega_{\mathrm{d}}> \\
(\mathbf{H z})\end{array}$ & $\begin{array}{c}\chi^{2} \\
\left(\mathbf{x} 10^{-4}\right) \\
\end{array}$ & $\begin{array}{c}\text { Width } \\
\text { (Hz) }\end{array}$ & $\begin{array}{l}\text { Relative } \\
\text { Width }\end{array}$ \\
\hline 0 & -127.30 & 2.92 & 1.36 & 128.05 & 636.05 & 70.94 & 135.34 & 3.65 & 113 & 0.83 \\
\hline 10 & -127.26 & 2.92 & 1.36 & 135.11 & 679.60 & 74.34 & 145.36 & 2.90 & 118 & 0.81 \\
\hline 50 & -127.10 & 3.54 & 1.53 & 136.28 & 652.19 & 69.25 & 145.36 & 3.54 & 110 & 0.76 \\
\hline 100 & -126.99 & 5.08 & 1.80 & 160.79 & 792.31 & 67.90 & 190.48 & 8.45 & 148 & 0.78 \\
\hline 250 & -126.49 & 7.49 & 2.26 & 178.17 & 765.84 & 62.45 & 205.51 & 8.21 & 181 & 0.88 \\
\hline
\end{tabular}

Table 2. SPME-GC/MS Peak Assignments

\begin{tabular}{cl}
\hline Elution Time (minutes) & Assignment \\
\hline 5.07 & Trimethyl silanol \\
5.43 & Tetrahydrofuran/Acetic Acid \\
6.91 & Trans-2(chlorovinyl) dimethylethoxysilane \\
8.23 & Cyclo-hexamethyltrisiloxane (d3) \\
8.60 & Butyrolactone \\
9.32 & Cyclo-octamethyltetrasiloxane (d4) \\
10.24 & Cyclo-decamethylpentasiloxane (d5) \\
11.31 & Cyclo-dodecamethylhexasiloxane (d6) \\
12.53 & Cyclo-tetradecamethylheptasiloxane (d7) \\
14.06 & Cyclo-hexadecamethyloctosiloxane (d8) \\
\hline
\end{tabular}


Figure 1. (A) $\chi^{2}$ as a function of input error in FTIKREG program. (B) Distributions of $<\Omega_{\mathrm{d}}>$ as a function of input error (and $\chi^{2}$ ) for pristine Sylgard 184. The color of the line changes from black to lighter gray with increasing error. The arrows in both figures indicate the chosen $\chi^{2}$ value and distribution curve, with the appropriate distribution curve shown in bold.
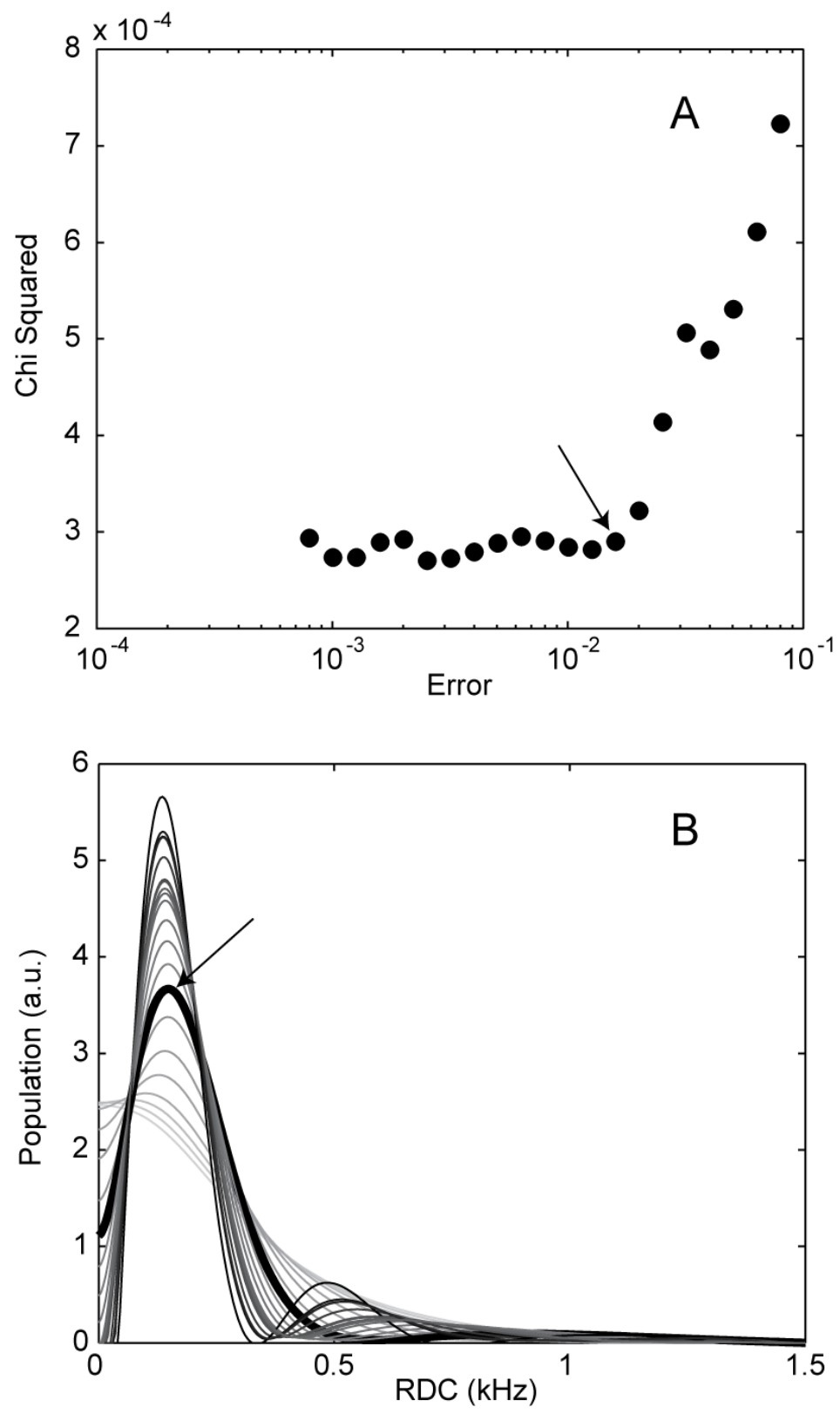
Figure 2. Charlesby-Pinner analysis via spin echo NMR for irradiated Sylgard 184.

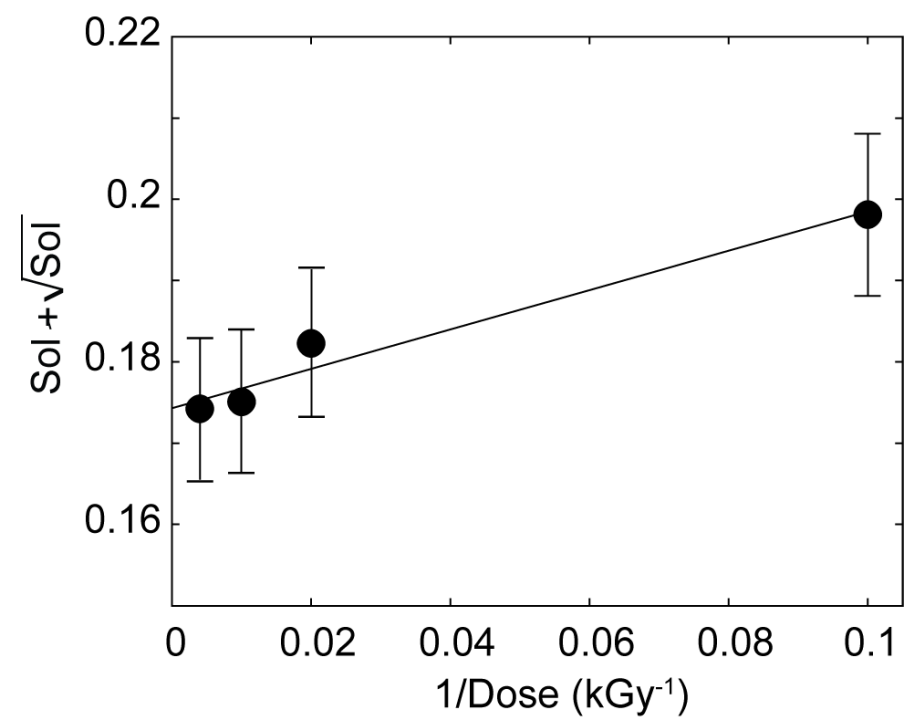


Figure 3. FTIKREG analysis of MQ growth curves for irradiated Sylgard 184.

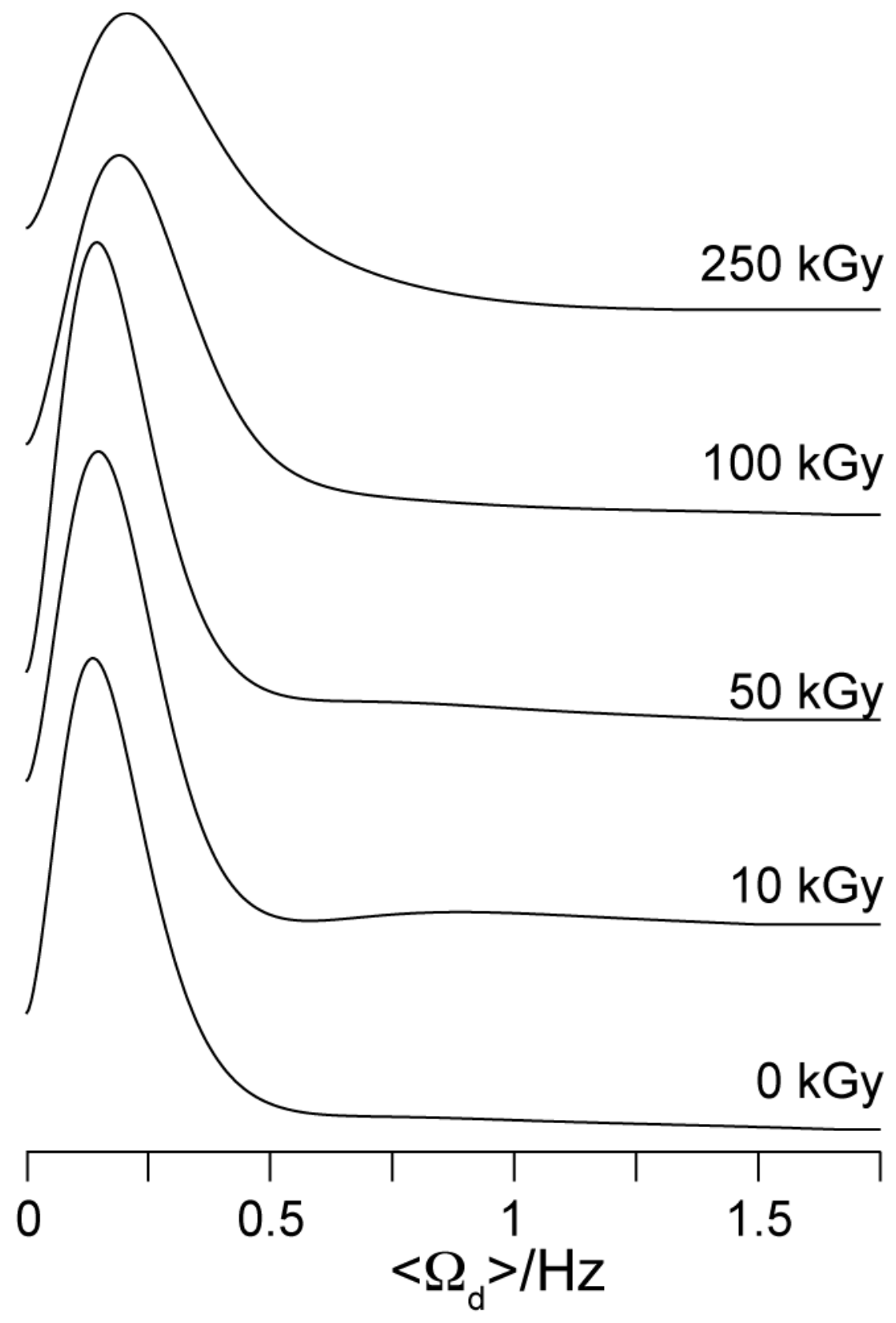


Figure 4. GC/MS total ion chromatograms from SPME headspace analysis of samples irradiated and held at room temperature (A) and aged at $70^{\circ} \mathrm{C}$ for two weeks (B).

A

$250 \mathrm{kGy}$
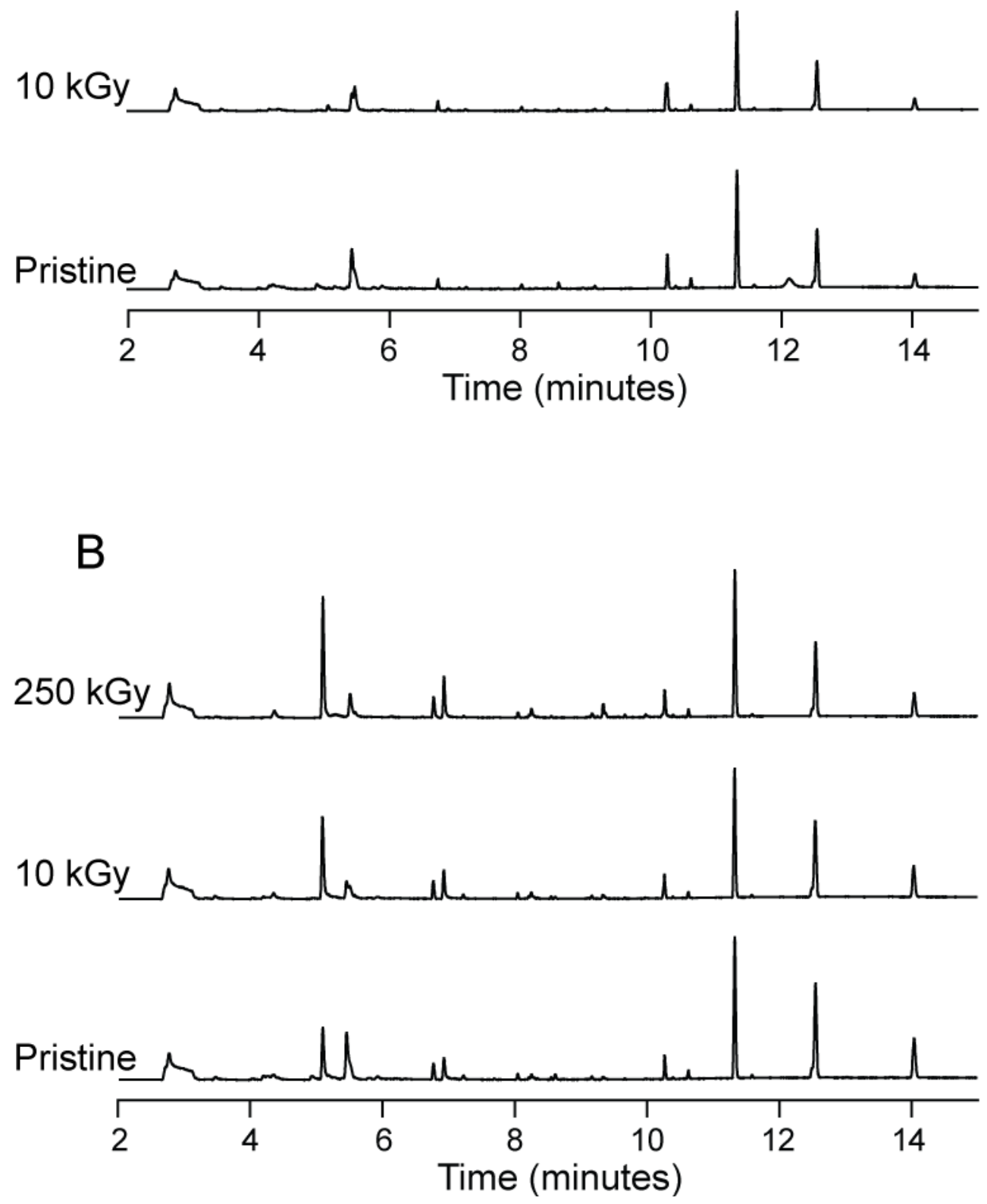
Figure 5. Principal Component Analysis of GC-MS data for samples irradiated and held at room temperature.

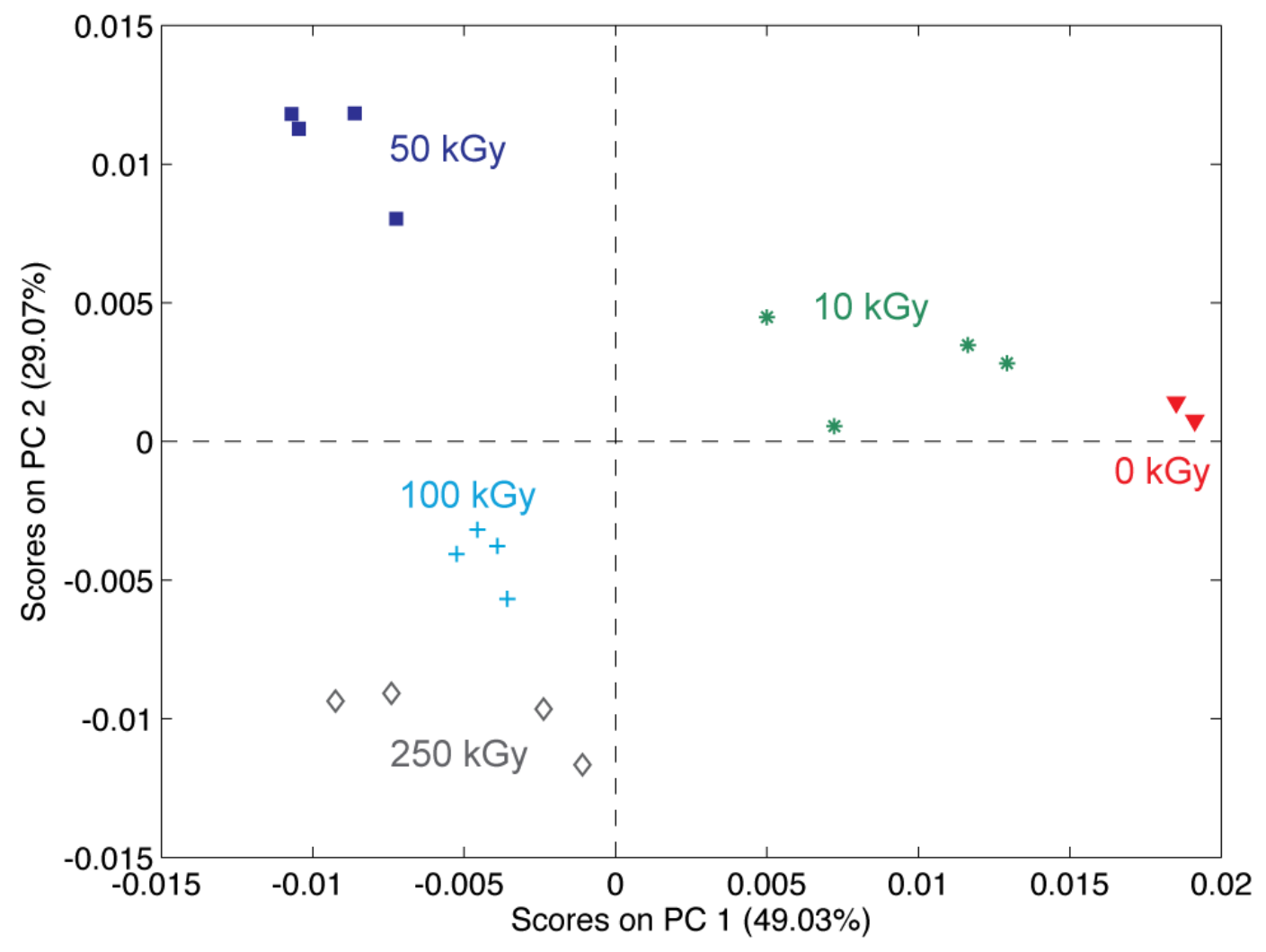


Figure 6. Scores (A) and loadings (B) plots for principal component 1.
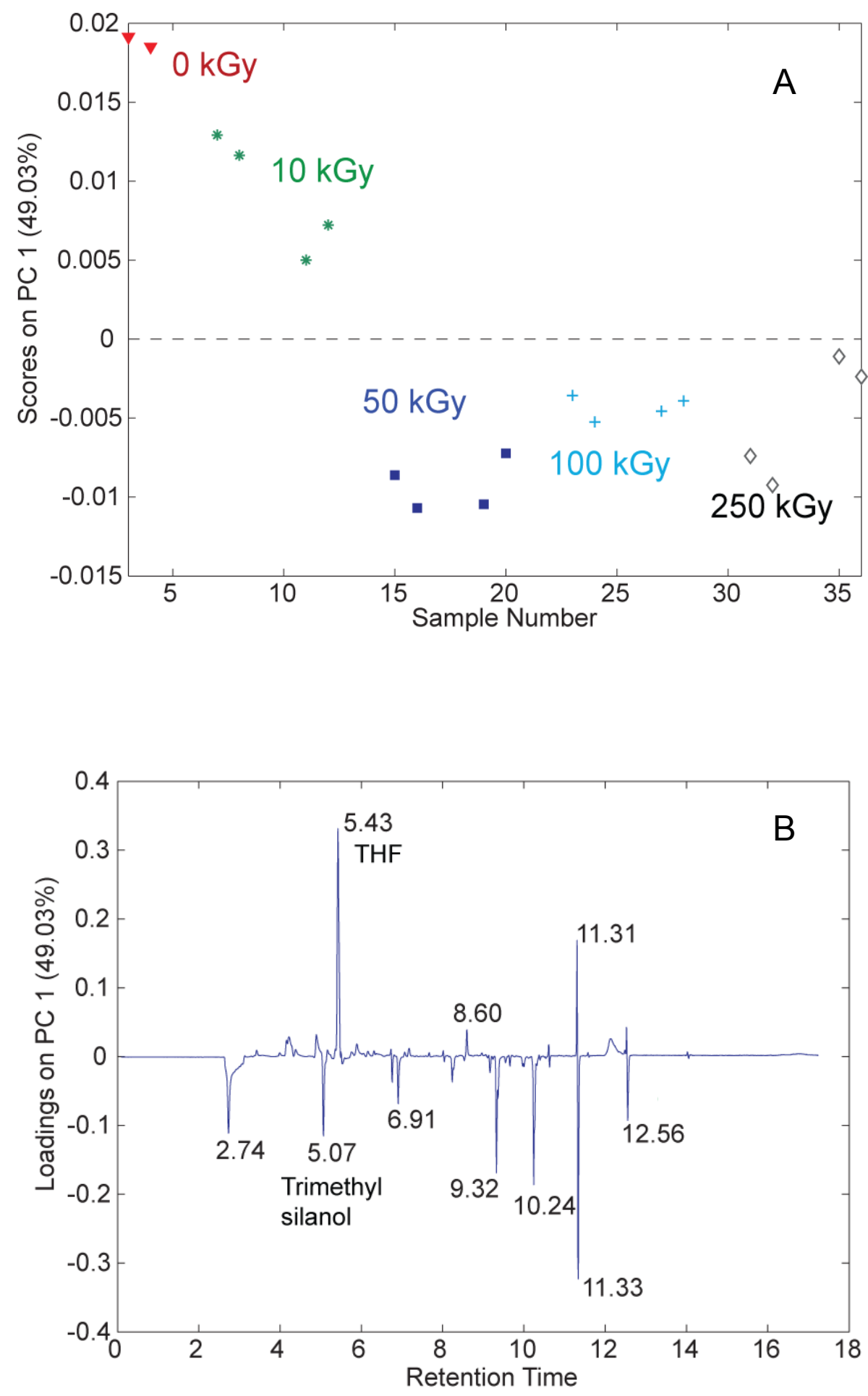
Figure 7. Scores (A) and loadings (B) plots for principal component 2.
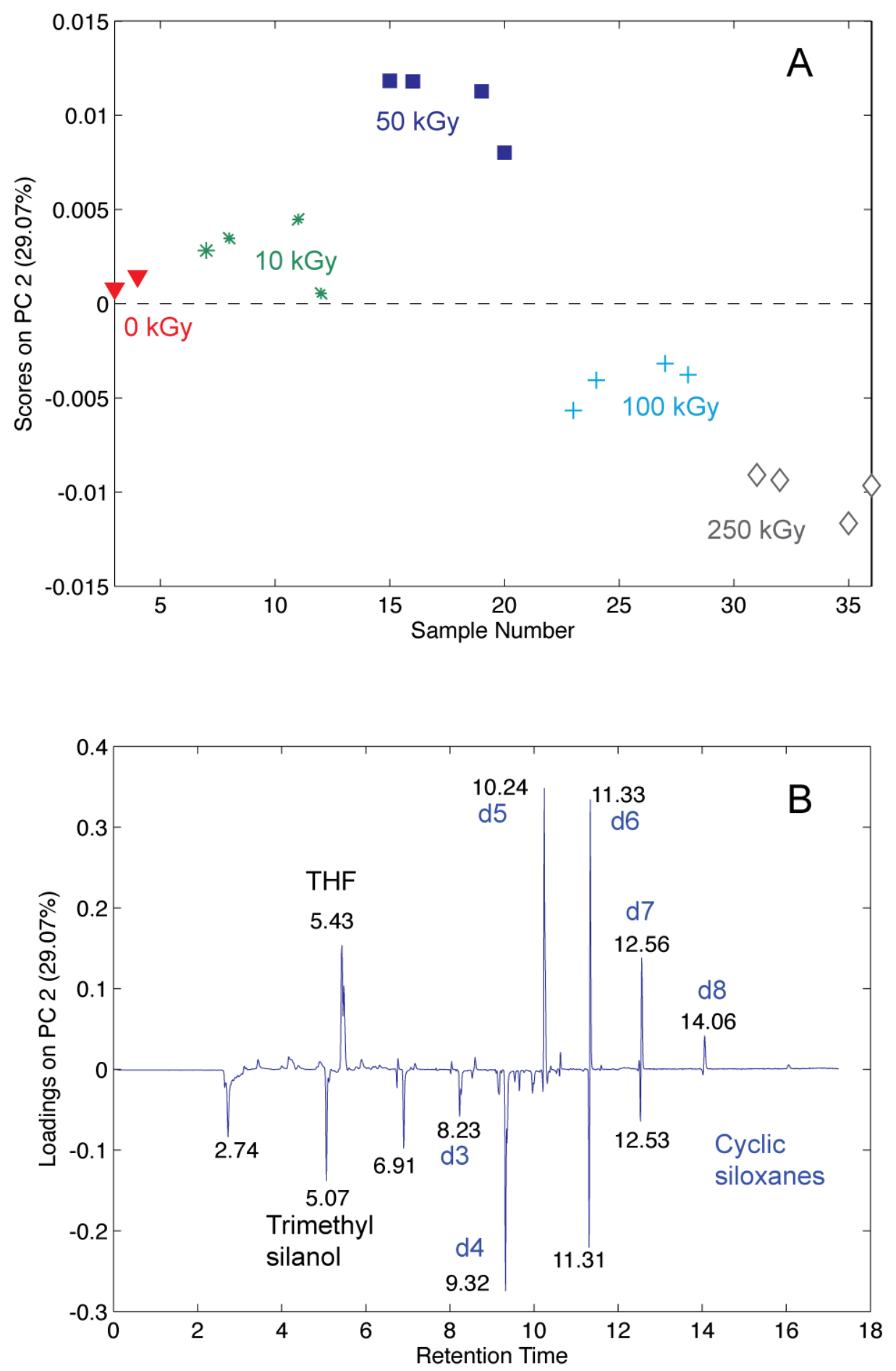
Figure 8. PCA plots comparing samples aged at $70^{\circ} \mathrm{C}$ vs. held at room temperature. (A) Scores plot for PC1 and PC2; (B) Loadings plot for PC1. Ovals are 90\% confidence ellipses.
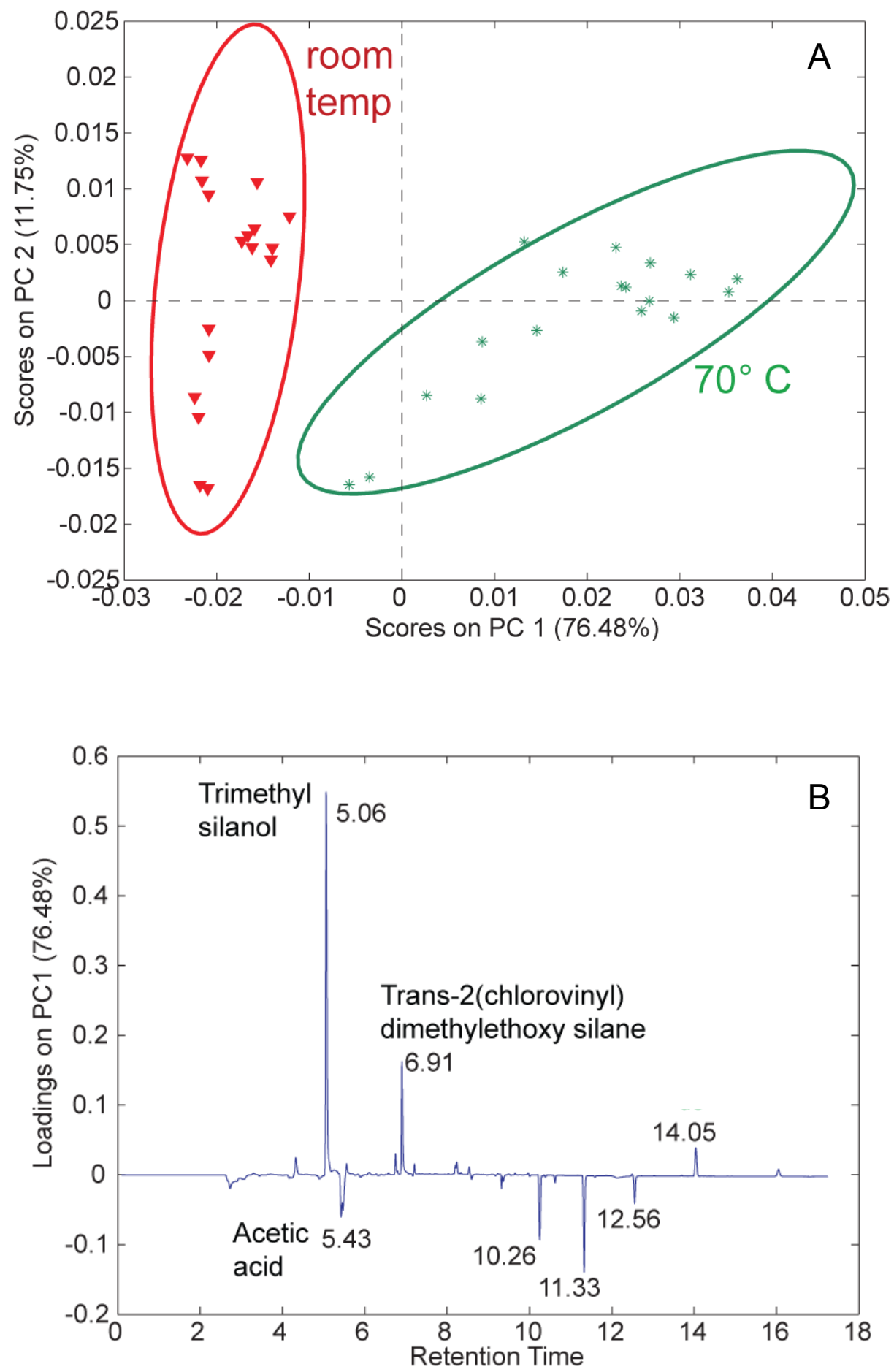
For Table of Contents Use Only:
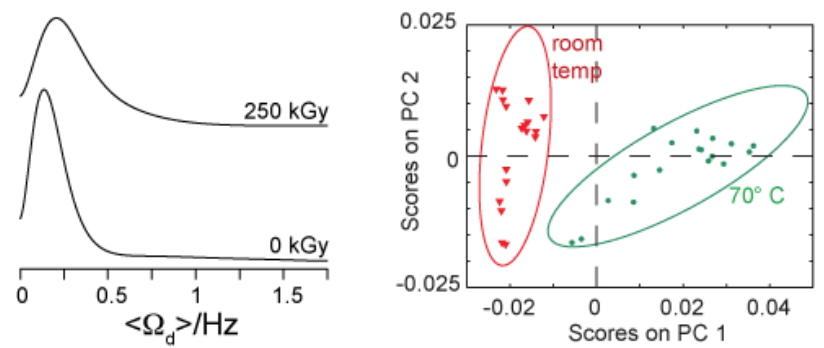